\title{
Landau theory of quantum spin glasses of rotors and Ising spins
}

\section{Citation}

Read, N., Subir Sachdev, and J. Ye. 1995. "Landau Theory of Quantum Spin Glasses of Rotors and Ising Spins." Physical Review B 52 (1): 384-410. https://doi.org/10.1103/physrevb.52.384.

\section{Permanent link}

http://nrs.harvard.edu/urn-3:HUL.InstRepos:41417429

\section{Terms of Use}

This article was downloaded from Harvard University's DASH repository, and is made available under the terms and conditions applicable to Other Posted Material, as set forth at http:// nrs.harvard.edu/urn-3:HUL.InstRepos:dash.current.terms-of-use\#LAA

\section{Share Your Story}

The Harvard community has made this article openly available.

Please share how this access benefits you. Submit a story.

\section{Accessibility}




\title{
Landau theory of quantum spin glasses of rotors and Ising spins
}

\author{
N. $\operatorname{Read}^{*}$ and Subir Sachdev ${ }^{\dagger}$ \\ Department of Physics, Yale University, \\ P.O. Box 208120, New Haven, CT 06520-8120 \\ and \\ Department of Applied Physics, Yale University, \\ P.O. Box 208284, New Haven, CT 06520-8284
}

Recent work on the zero-temperature phase transition in a quantum spin glass of rotors or Ising spins is reviewed.

\section{INTRODUCTION}

This paper will be a review of our recent work, done in collaboration with J. Ye [1] and R. Oppermann [2]. We consider some of the simplest quantum generalizations of classical spin glass problems, in which, for the sake of definiteness, the Hamiltonian can be taken to be one of the following: (i) The Hamiltonian $\mathcal{H}_{I}$ of the Ising model in a transverse field:

$\mathcal{H}_{I}=-g \sum_{i} \sigma_{i}^{x}-\sum_{<i j>} J_{i j} \sigma_{i}^{z} \sigma_{j}^{z}$

Here $\sigma^{x}, \sigma^{z}$ are the $x, z$ components of the three Pauli spin operators, with the Pauli operators on different sites commuting with each other. Each site, therefore, has an Ising degree of freedom, represented by the eigenvalues of the $\sigma_{i}^{z}$. The $\sigma_{i}^{x}$ is the kinetic energy term (due to the transverse magnetic field) and induces on-site flips of the Ising spins. (ii) The Hamiltonian $\mathcal{H}_{R}$ of quantum rotors:

$\mathcal{H}_{R}=\frac{g}{2} \sum_{i} \hat{\mathbf{L}}_{i}^{2}-\sum_{\langle i j\rangle} J_{i j} \hat{\mathbf{n}}_{i} \cdot \hat{\mathbf{n}}_{j}$

Here, the $M$-component vectors $\hat{\mathbf{n}}_{i}$, with $M \geq 2$, are of unit length, $\hat{\mathbf{n}}_{i}^{2}=1$, and represent the orientation of the rotors on the surface of a sphere in $M$-dimensional rotor space. The operators $\hat{L}_{i \mu \nu}$ $(\mu<\nu, \mu, \nu=1 \ldots M)$ are the $M(M-1) / 2$ components of the angular momentum $\hat{\mathbf{L}}_{i}$ of the

\footnotetext{
*Research supported by NSF grant no. DMR-91-57484.
}

${ }^{\dagger}$ Research supported by NSF grant no. DMR-92-24290. rotor; the first term in $\mathcal{H}_{R}$ is the kinetic energy of the rotor with $1 / \mathrm{g}$ the moment of inertia. The different components of $\hat{n}_{i}$ constitute a complete set of commuting observables and the state of the system can be described by a wavefunction $\Psi\left(n_{i}\right)$. In either model, the sum $\langle i j\rangle$ is over nearest. neighbors sites on a hypercubic $d$-dimensional lattice, and the $J_{i j}$ 's are drawn independently with a common Gaussian probability distribution of mean zero and variance $\left[J_{i j}^{2}\right]=J^{2}$ (throughout, the square brackets [...] will denote the average with respect to this distribution). Model (i) can be considered as the $M=1$ case of model (ii), and the models have global $O(M)$ symmetry (note that $\left.O(1) \cong Z_{2}\right)$. We introduce a unified notation $S_{i \mu}$, with $M \geq 1$, such that

$S_{i \mu}=\left\{\begin{array}{l}\hat{n}_{i \mu} \text { for } M \geq 2 \\ \sigma_{i}^{z} \text { for } M=1 .\end{array}\right.$,

which will be useful for writing the correlation functions later

We have also studied a model of a metallic quantum spin glass [2]. Some differences of the properties of this model from those of models (i) and (ii) will be described in Section 6 .

In quantum or classical statistical systems with quenched disorder, the thermodynamic quantities and correlation functions, etc, generally depend on the random variables in the Hamiltonian (here $J_{i j}$ ), and so are themselves random variables. The goal is to find averages over the disorder (or higher moments, or even distribution functions) of thesc cxperimentally relevant quanti- 
ties. This procedure defines the term "quenched" which was used above. For this purpose, the socalled replica method is often employed, and will be used herein.

Spin glass and spin-glass-like problems are defined by the property that the natural order parameter, that distinguishes the ordered phase (where the order parameter is nonzero) from the non-ordered phase (where it is zero), and which for our models would be $\left\langle S_{i \mu}\right\rangle$, is a random variable with zero mean, and one must consider the variance, $\left[\left\langle S_{i \mu}\right\rangle^{2}\right]$, in order to find a quantity that does distinguish the two phases of the system. This is known as the Edwards- Anderson order parameter $q_{E A}$. (Throughout, angle brackets $\langle\ldots\rangle$ will denote quantum and/or thermal averages using the Hamiltonian, such as that of models (i) or (ii), which depends on the random variables.) Quantum spin glass problems are low temperature spin-glass-like problems where quantum mechanics plays a significant role, especially when the transition occurs at zero temperature, by varying some parameter such as $g / J$ in our models. Part of the motivation for studying such problems comes from experiments. Experimentally studied systems include magnetic systems where the application of the "spin" language used here is fairly direct; these include a transverse field Ising system [3], the high $T_{c}$ system $\mathrm{La}_{2-x} \mathrm{Sr}_{x} \mathrm{CuO}_{4}$ at small $x$, and the "heavy fermion" compound $Y_{1-x} U_{x} P d_{3}$ [4]. (Some of these systems may require the use of Heisenberg spins in which, unlike the models considered here, the components of the spin operators $S_{i \mu}$ obey the commutation relations of angular momentum at each site. These models have been considered in [5], where the paramagnetic phase is studied.) Other physical problems having spin-glass-like aspects include metalinsulator transitions, superfluid-insulator transitions, and the transitions between quantum Hall plateaus. Thus quantum problems with randomness, in general, and spin-glass-like problems, in particular, are of considerable interest in condensed matter physics.

We next describe the expected phase diagrams for our models. In sufficiently high dimensions, we expect a $T / J$ versus $g / J$ phase diagram like

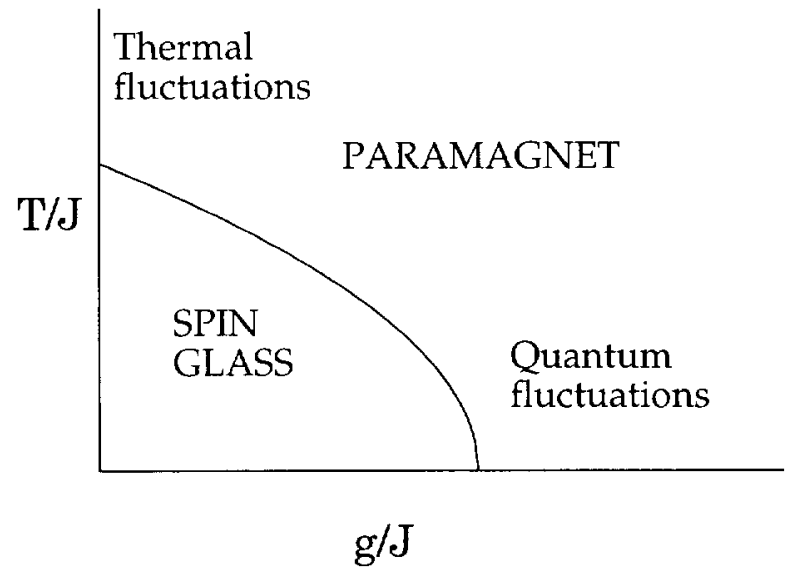

Figure 1.

that in Fig. 1. Here $g / J$ is a dimensionless parameter describing the relative strength of quantum fluctuations. The phases at zero temperature can be understood by considering the limits $J=0$ and $g=0$. In the limit $J=0$ (where all $J_{i j}=0$ ), both $\mathcal{H}_{R}$ and $\mathcal{H}_{I}$ possess non-degenerate ground states which preserve the global symmetry. For the rotors, each site is in the spherically symmetric ' $s$-wave' state (using the language of $M=3$ ). Similarly, each Ising spin is in the eigenstate $\left(\left|\sigma^{z}=+1\right\rangle+\left|\sigma^{z}=-1\right\rangle\right) / \sqrt{2}$ of $\sigma^{x}$ with eigenvalue +1 and so is $\mathbf{Z}_{2}$ invariant $\left(\mathbf{Z}_{2}\right.$ symmetry is in fact implemented by the action of $\left.\prod_{i} \sigma_{i}^{x}\right)$. In both cases, the $J_{i j}=0$ ground state is separated from the first excited state by a gap of order $g$. It is therefore reasonable to consider the $J_{i j}$ 's as perturbations in this limit (though the random nature of the $J_{i j}$ causes some problems which will not be discussed here) and to expect that this "quantum- disordered" or paramagnetic phase persists at finite values of $J$. In the opposite limit, where $g$ is zero, the Hamiltonian reduces to that of a classical spin glass. The interaction terms proportional to the $J_{i j}$ would prefer a ground state in which each rotor or Ising spin has a definite orientation which minimizes the exchange energy, breaking $O(M)$ symmetry, and quantum fluctuations are absent; this state is the ground state of the classical spin glass. A small $g$ will not necessarily destroy this phase, in sufficiently high spatial dimension. We therefore expect that both ordered and disordered phases exist at zero temperature, at least in sufficiently 
high dimensions $d$. At finite temperature, the question of the existence of a phase transition from paramagnet to spin glass can be settled classically, by standard arguments, and moreover, if there is a transition on some phase boundary as indicated in Fig. 1, its universality class will be the same as that of the classical spin glass model for all $T>0$. The transition point on the zero temperature axis, however, will be in a distinct universality class because quantum fluctuations cannot be ignored (at least, not for any obvious reason). It is the vicinity of this point which is our main object of study. For the classical model, there will be no transition when the dimension $d$ is less than some lower critical dimension for the classical problem, $d<d_{l}^{\text {class }}$. However the zero temperature quantum transition probably survives down to a lower dimension $d_{l}^{\text {qu }}$. In the range $d_{l}^{\text {qu }}<d<d_{l}^{\text {class }}$, the phase diagram has a transition point on the zero temperature axis, but no phase boundary at finite $T$ entering it. However, the existence of a transition in the ground state is still revealed by the difference between the order parameters in the ground states on either side.

A phase diagram of the same form as in Fig. 1 is in fact obtained exactly within an infinite range version of the spin glass models in the $M \rightarrow \infty$ limit [7]. The infinite range models provide a mean field description of the critical phenomena, just as they do in the classical case. In the quantum case, however, these models cannot be solved exactly in general, but their critical properties can be obtained $[6,7]$. The approach [1] we describe here complements and extends the methods used in those papers; it provides, by the use of a Landau theory: a simple view of the exact mean field critical exponents and universal crossover functions obtained in $[6,7]$. The Landau theory involves an expansion in powers of the order parameter, momenta and frequencies, and so is expected to be valid at most for the universal long-distance, long time properties near the zero-temperature critical point.

\section{LANDAU THEORY}

We begin by sketching an explicit derivation of the Landau effective action from a microscopic model similar to models (i) and (ii). It is slightly more convenient to work with soft spins rather than the fixed-length quantum rotors or Ising spins (although the derivation below can be extended to these cases). We begin with the pathintegral for these spins in the presence of a fixed realization of the disorder

$$
\begin{aligned}
Z= & \int \mathcal{D} S_{i \mu} \exp \left(-\int d \tau\left\{\mathcal{L}_{0}\left(S_{i \mu}\right)\right.\right. \\
& \left.\left.-\sum_{<i j>} J_{i j} S_{i \mu} S_{j \mu}\right\}\right) \\
\mathcal{L}_{0}= & \sum_{i}\left[\frac{1}{2 g}\left(\partial_{\tau} S_{i \mu}\right)^{2}+\frac{m^{2}}{2} S_{i \mu}^{2}+\frac{\tilde{u}}{2}\left(S_{i \mu}^{2}\right)^{2}\right] .
\end{aligned}
$$

This action may be interpreted as that of $M$ component harmonic oscillators on the sites $i$ of a $d$-dimensional lattice, with a non-linear selfcoupling $\tilde{u}$ and a random nearest neighbor interaction $J_{i j}$. We now introduce replicas (indexed by $a=1, \ldots, n$ with $n \rightarrow 0$ ) and average over a Gaussian distribution of the $J_{i j}$. We obtain the replicated, translationally invariant result:

$$
\begin{aligned}
{\left[Z^{n}\right]=} & \int \mathcal{D} S_{i \mu}^{a} \exp \left(-\int d \tau \sum_{a} \mathcal{L}_{0}\left(S_{i \mu}^{a}\right)\right. \\
& -\frac{J^{2}}{2} \sum_{<i j>} \int d \tau_{1} d \tau_{2} \sum_{a b} S_{i \mu}^{a}\left(\tau_{1}\right) S_{j \mu}^{a}\left(\tau_{1}\right) \\
& \left.S_{i \mu}^{b}\left(\tau_{2}\right) S_{j \mu}^{b}\left(\tau_{2}\right)\right)
\end{aligned}
$$

Now, as in classical spin glasses [8], we decouple the quartic term by a Hubbard-Stratonovich transformation:

$$
\begin{aligned}
{\left[Z^{n}\right]=} & \int \mathcal{D} Q_{i \mu \nu}^{a b} \exp \left(-\frac{J^{2}}{2} \int d \tau_{1} d \tau_{2}\right. \\
& \sum_{i, j} \sum_{a b} Q_{i \mu \nu}^{a b}\left(\tau_{1}, \tau_{2}\right) K_{i j}^{-1} Q_{j \mu \nu}^{a b}\left(\tau_{1}, \tau_{2}\right) \\
& \left.+\ln Z_{S}[Q]\right)
\end{aligned}
$$




$$
\begin{aligned}
Z_{S}[Q]= & \int \mathcal{D} S_{i \mu}^{a} \exp \left(-\int d \tau \sum_{a} \mathcal{L}_{0}\left(S_{i \mu}^{a}\right)\right. \\
& -\int d \tau_{1} d \tau_{2} \sum_{i} \sum_{a b} Q_{i \mu \nu}^{a b}\left(\tau_{1}, \tau_{2}\right) \\
& \left.S_{i \mu}^{a}\left(\tau_{1}\right) S_{i \nu}^{b}\left(\tau_{2}\right)\right)
\end{aligned}
$$

where $K_{i j}$ is the incidence matrix of the lattice, equal to 1 when $i, j$ are nearest neighbors, 0 otherwise (including $i=j$ ). Here we see a distinguishing feature of the quantum spin glass problem: $Q$ is a function of two (imaginary) times, due to the randomness being time-independent. We now expand $\ln Z_{S}[Q]$ in powers, not of $Q_{\mu \nu}^{a b}$ itself, but of $Q_{\mu \nu}^{a b}\left(x, \tau_{1}, \tau_{2}\right)-C \delta_{\mu \nu}^{a b} \delta\left(\tau_{1}-\tau_{2}\right)$ for a suitably chosen value of $C$, to be explained below; the constant $C$ can then be absorbed into $\mathcal{L}_{0}$ and becomes part of the quadratic $S_{i \mu}^{2}$ term, which can be shown to remain stable at small $\tilde{u}$. At the same time, we keep only the terms with few derivatives with respect to time or space.

This procedure yields the Landau functional (recall that we are using the Einstein summation convention for the $\mathrm{O}(M)$ vector indices):

$$
\begin{aligned}
\mathcal{A}= & \frac{1}{t} \int d^{d} x\left\{\frac{1}{\kappa} \int d \tau \sum_{a}\right. \\
& {\left.\left[\frac{\partial}{\partial \tau_{1}} \frac{\partial}{\partial \tau_{2}}+r\right] Q_{\mu \mu}^{a a}\left(x, \tau_{1}, \tau_{2}\right)\right|_{\tau_{1}=\tau_{2}=\tau} } \\
& +\frac{1}{2} \int d \tau_{1} d \tau_{2} \sum_{a, b}\left[\nabla Q_{\mu \nu}^{a b}\left(x, \tau_{1}, \tau_{2}\right)\right]^{2} \\
& -\frac{\kappa}{3} \int d \tau_{1} d \tau_{2} d \tau_{3} \sum_{a, b, c} Q_{\mu \nu}^{a b}\left(x, \tau_{1}, \tau_{2}\right) \\
& Q_{\nu \rho}^{b c}\left(x, \tau_{2}, \tau_{3}\right) Q_{\rho \mu}^{c a}\left(x, \tau_{3}, \tau_{1}\right) \\
& +\frac{1}{2} \int d \tau \sum_{a}\left[u Q_{\mu \nu}^{a a}(x, \tau, \tau) Q_{\mu \nu}^{a a}(x, \tau, \tau)\right. \\
& \left.\left.+v Q_{\mu \mu}^{a a}(x, \tau, \tau) Q_{\nu \nu}^{a a}(x, \tau, \tau)\right]\right\} \\
& -\frac{1}{2 t^{2}} \int d^{d} x \int d \tau_{1} d \tau_{2} \sum_{a, b} Q_{\mu \mu}^{a a}\left(x, \tau_{1}, \tau_{1}\right) \\
& Q_{\nu \nu}^{b b}\left(x, \tau_{2}, \tau_{2}\right)+\cdots
\end{aligned}
$$

There are four terms in $\mathcal{A}$ whose co-efficients contain products of powers of only two coupling con- stants, $\kappa$ and $t$; this form can be reached without loss of generality by suitably rescaling the space and time co-ordinates. The reasons for our rather peculiar choices for these couplings will only become evident when the structure of perturbation theory is discussed. We have only retained the terms which our later power counting will tell us are relevant or marginal in high space dimension $d$, together with the leading irrelevant term. The exception to this statement is a quadratic term

$\int d^{d} x d \tau_{1} d \tau_{2} \sum_{a, b}\left[Q_{\mu \nu}^{a b}\left(x, \tau_{1}, \tau_{2}\right)\right]^{2}$

which appears to be highly relevant in all $d$. However, it is a "redundant" operator as it can be removed by a further transformation $Q \rightarrow$ $Q-C \delta^{a b} \delta_{\mu \nu} \delta\left(\tau_{1}-\tau_{2}\right)$ for a suitable choice of $C$. This relies on the presence of the cubic term with coefficient $\kappa / t$. Thus, the constant $C$ can be chosen such that, if $r$ is redefined to absorb a constant, the action has the form given in (7). We will see that this choice of definition of $Q$ to eliminate the term in $(8)$ also makes $\langle\langle Q\rangle\rangle=0$ at $\omega=0$ and $g=g_{c}$. (We have introduced double angular brackets to represent averages taken with the translationally invariant replica action.) It follows that $\langle\langle Q\rangle\rangle$ will be small for $g \approx g_{c}$ and $\omega \approx 0$, so that the Landau theory expansion of $\mathcal{A}$ in powers of $Q$ is valid. This leaves $r$, the coupling in the term linear in $Q$, as the parameter expected to drive the system through its transition.

A few last remarks on the action $\mathcal{A}$ : The rather unfamiliar looking time derivatives in the linear term can be seen to follow from a gradient expansion of a term like $\int d^{d} x d \tau_{1} d \tau_{2} Q_{\mu \mu}^{a a}\left(x, \tau_{1}, \tau_{2}\right) f\left(\tau_{1}-\tau_{2}\right)$ where $f(\tau)$ is an even function of $\tau$ which falls rapidly to zero within a $\tau$ of order $1 / g$. All the terms in $\mathcal{A}$ are generated by the expansion of $\ln Z_{S}[Q]$, with the exception of the last $1 / t^{2}$ term. There are two routes to generating such a term:

(i) Renormalize the functional integral over $Q$ itself, by diagrammatic perturbation theory in $Q$. At order $\kappa^{2}$ one finds a Feynman diagram that generates a term of the form of the final term in $\mathcal{A}$. Thus, even though it is absent in the bare $Q$ action, it will eventually appear. For the renormalization group analysis later, it is therefore ad- 
vantageous to include the $1 / t^{2}$ term at the starting point.

(ii) Introduce additional on-site sources of randomness in $\mathcal{L}_{0}$. Randomness in the value of $m^{2}$ couples to the $\left[S_{i \mu}^{a}(\tau)\right]^{2}$, an operator which is essentially the same as $Q^{a a}(x, \tau, \tau)$. Integrating over the randomness will then generate the $1 / t^{2}$ term. Randomness in $g$ or $\tilde{u}$ has the same effect. The terms with coefficients $u / t$ and $v / t$ arise from quartic couplings of the spins within a single replica. They are the only terms retained that break replica $O(n)$ symmetry to $S_{n}$ permutational symmetry. If they were omitted, the action would describe randomly coupled simple harmonic oscillators, which is definitely an unstable system in finite dimensions, and so anharmonic terms (and hence $u$ and $v$ ) are expected to be necessary for stability.

Finally note that, apart from the delta-function term at short times, $Q$ represents in the long wavelength theory

$Q_{\mu \nu}^{a b}\left(x, \tau_{1}, \tau_{2}\right)=\sum_{i \in \mathcal{N}(x)} S_{i \mu}^{a}\left(\tau_{1}\right) S_{i \nu}^{b}\left(\tau_{2}\right)$

where $\mathcal{N}(x)$ is a coarse-graining region in the neighborhood of $x$. This will be useful in interpreting the theory.

\section{MEAN FIELD THEORY}

Given the action, the next step is to find an extremum, as a means to approximate the path integral by the saddle point method. The extremum constitutes the mean field approximation for the problem. This will then form the starting point for the analysis of fluctuations around the mean field theory, which will require the use of renormalization group techniques in general.

The mean field approximation is more accurately called the tree approximation, i.e. there are no momentum loop integrations for fluctuations. For the infinite range model, where spatial dependence of $Q$ can be dropped to obtain either thermodynamic quantities as averages over the whole system, or onsite correlation functions, this is exact and constitutes a simpler rederivation of results obtained earlier [7]. For the short-range finite dimensional quantum models, as in the clas- sical case [8], mean-field theory should be a useful starting point towards understanding the overall phase diagram and properties of the phases. For the critical properties of the quantum transition, the mean-field theory is an approximation whose validity as an attractive weak-coupling fixed point under the renormalization group in sufficiently high dimensions will be examined later. Here we will concentrate on the correlators, and study only the paramagnetic phase and critical point.

The saddle-point and perturbative analysis are most conveniently performed in momentum $(k)$ and frequency $(\omega)$ space. We will work at a finite, but small, temperature $T=1 / \beta$, and $\omega$ will therefore take values at the discrete Matsubara frequencies. The normalization of the Fourier transform is set by

$$
\begin{aligned}
& Q\left(k, \omega_{1}, \omega_{2}\right)= \\
& \quad \int d^{d} x \int_{0}^{\beta} d \tau_{1} d \tau_{2} Q\left(x, \tau_{1}, \tau_{2}\right) e^{i\left(k x-\omega_{1} \tau_{1}-\omega_{2} \tau_{2}\right)} .
\end{aligned}
$$

In these Fourier transformed variables we expect the saddle point-value of $Q$ to obey the following ansatz in the paramagnet and at the critical point

$$
\begin{aligned}
& Q_{\mu \nu}^{a b}\left(k, \omega_{1}, \omega_{2}\right)= \\
& \quad \beta \hat{\delta}_{\mu \nu} \delta^{a b}(2 \pi)^{d} \delta^{d}(k) \delta_{\omega_{1}+\omega_{2}, 0} D\left(\omega_{1}\right) .
\end{aligned}
$$

An explicit factor of $\beta$ has been inserted to make $D(\omega)$ finite in the zero temperature limit. The structure in the $O(M)$ spin space follows from spin rotation invariance, while the replicadiagonal structure follows from the absence of a static moment in the paramagnetic phase. Inserting this into $\mathcal{A}$ in (7), we obtain for the free energy density $\mathcal{F} / n$ (as usual, $\mathcal{F} / n$ represents the physical disorder-averaged free energy)

$$
\begin{gathered}
\frac{\mathcal{F}}{n}=\frac{M}{\beta t} \sum_{\omega}\left[\frac{\omega^{2}+r}{\kappa} D(\omega)-\frac{\kappa}{3} D^{3}(\omega)\right] \\
+M \frac{u+M v}{2 t}\left[\frac{1}{\beta} \sum_{\omega} D(\omega)\right]^{2} .
\end{gathered}
$$

The contribution from the last $1 / t^{2}$ term in $\mathcal{A}$ vanishes in the replica limit $n \rightarrow 0$ and is therefore absent. The stationary point with respect to 
variations in $D(\omega)$ gives us the result

$D(\omega)=-\frac{1}{\kappa}\left(\omega^{2}+\tilde{r}\right)^{1 / 2}$

where $\tilde{r}$ is given implicitly by

$\tilde{r}=r-(u+M v) \frac{1}{\beta} \sum_{\omega}\left(\omega^{2}+\tilde{r}\right)^{1 / 2}$.

The sign of $D(\omega)$ is determined by the fact that the Fourier transform $D(\tau)$ is positive. This solution for $D(\omega)$ is well-defined for $\tilde{r} \geq 0$, while no sensible paramagnetic solution exists for $\tilde{r}<0$, suggesting that the critical line in the $r, T$ plane between the paramagnetic and spin glass phases is $\tilde{r}=0$. The local density of excitations $\chi^{\prime \prime}(\omega)$ can be obtained by analytic continuation of $D(\omega)$ to real frequencies and is therefore

$\chi^{\prime \prime}(\omega)=\operatorname{sgn}(\omega) \frac{\left(\omega^{2}-\tilde{r}\right)^{1 / 2}}{\kappa} \theta(|\omega|-\sqrt{\tilde{r}})$.

There is a gap, $\Delta=\sqrt{\tilde{r}}$, in the spectral density which vanishes at the critical point $\tilde{r}=0$. This gap is expected to be filled in at finite temperatures by loop corrections involving inelastic effects; in addition, Griffiths effects will lead to sub- gap absorption at both zero and finite temperatures.

¿From (14) we determine that the critical point $\tilde{r}=0$ occurs when

$r=r_{c}(T) \equiv \frac{u+M v}{\beta} \sum_{\omega}|\omega|$.

The frequency summation is obviously divergent, and the result will depend upon the nature of the ultraviolet cutoff. However the temperature dependence of the result is entirely in the subleading term, which turns out to be cutoff-independent (provided the cutoff is smooth on the scale of $T$ ). The summation can be evaluated by the Poisson summation formula, which yields:

$r_{c}(T)=r_{c}-(u+M v) \frac{\pi T^{2}}{3}$

where for a high-frequency cutoff around $\Lambda_{\omega} \sim g$

$r_{c} \equiv r_{c}(0)=\frac{(u+M v) \Lambda_{\omega}^{2}}{2 \pi}$.
The line between the paramagnetic and spin-glass phases is shown in Fig. 1 in the $r, T$ (or $g, T$ ) plane.

The behavior of the "gap" parameter $\tilde{r}$ close to $r=r_{c}$ and at finite $T$, is given by

$\tilde{r}= \begin{cases}\frac{4 \pi}{(u+M v)} \frac{\left(r-r_{c}\right)}{\log \left(\Lambda_{\omega}^{2} /\left(r-r_{c}\right)\right)} & \text { I } \\ \frac{2 \pi^{2} T^{2}}{3 \log \left(\Lambda_{\omega} / T\right)} & \text { II } \\ \frac{\left(r-r_{c}(T)\right)^{2}}{T^{2}} & \text { III, }\end{cases}$

in three regimes I, II, III, characterized as follows. Regime I, where $\left(r-r_{c}\right)^{1 / 2} \gg T$, is that of the quantum paramagnet, where the properties are those of the quantum- disordered ground state, and thermal effects are not important. Regime II, where $T \gg\left|r-r_{c}\right|^{1 / 2}$, is "quantum-critical": here the system behaves as if it is at the critical point $r=r_{c}$, and the properties reflect those of the critical ground state and its excitations; it is the analog of the quantum-critical region discussed in Refs $[9,10]$ for quantum rotors in the absence of disorder. Finally, in regime III, close enough to the finite- temperature phase transition, where $\left(r-r_{c}(T)\right)^{1 / 2} \ll T$, classical effects take over completely, and the behavior is that of the usual finite-temperature spin- glass/paramagnet transition in the classical model [8]. Regime III also extends into the spin glass phase, although here we have only obtained results for its paramagnetic portion. Notice that in regime III, $\tilde{r}$ now depends upon the square of the distance from the transition $r-r_{c}(T)$ : as will become clear from later results, $\tilde{r}^{1 / 4}$ plays the role of an inverse correlation length, so this is just what is needed to transform the quantum model with $\nu=1 / 4$ to the classical model with $\nu=1 / 2$ in mean-field theory. The results (15) and (19) for the local spectral weight and the asymptotic form of the gap, including the logarithmic correction, are identical to those obtained in the infinite range model by different methods earlier $[6,7]$. The reason for the logarithmic corrections, which are unusual in a mean field theory, will be clarified in the context of RG later; here we need only mention that they result from the $u$ and $v$ terms which induce a frequency sum, even in mean field theory. 
$D$ represents the disorder average

$D\left(\tau_{1}-\tau_{2}\right)=\frac{1}{M}\left[\left\langle S_{i \mu}\left(\tau_{1}\right) S_{i \mu}\left(\tau_{2}\right)\right\rangle\right]$

in the original random problem. At $r>r_{c}(g>$ $\left.g_{c}\right), D$ decays exponentially with $\tau$ as $\tau \rightarrow \infty$, due to the gap $\Delta=\sqrt{\tilde{r}}$ in the corresponding spectral density; at $g=g_{c}, D$ decays as $1 / \tau^{2}$, and in the ordered phase, $D \rightarrow$ constant $=q_{E A}$. Thus from the behavior of the gap $\Delta$, we can define an exponent $z \nu$, anticipating anisotropic scaling in space and time, which takes the value $z \nu=1 / 2$ in the infinite range model. Since $D(\tau \rightarrow \infty)$ is the Edwards-Anderson order parameter, we may also define an exponent $\beta$ by $q_{E A} \sim\left(g_{c}-g\right)^{\beta}$ and it is found that $\beta=1$. At $g=g_{c}$ one expects $D(\tau) \sim \tau^{-\beta / z \nu}$ which is satisfied with the values already obtained.

A quantity intimately related to the spin glass long range order is the quantum mechanically disconnected correlation function

$$
\begin{aligned}
& G\left(i-j, \tau_{1}-\tau_{2}, \tau_{3}-\tau_{4}\right) \equiv \\
& \quad\left[\left\langle S_{i \mu}\left(\tau_{1}\right) S_{j \mu}\left(\tau_{2}\right)\right\rangle\left\langle S_{i \nu}\left(\tau_{3}\right) S_{j \nu}\left(\tau_{4}\right)\right\rangle\right] .
\end{aligned}
$$

Note that $\left[\left\langle S_{i \mu}\left(\tau_{1}\right) S_{j \mu}\left(\tau_{2}\right)\right\rangle\right]=0$ for $i \neq j$ because of the $Z_{2}$ gauge symmetry, and no subtraction of products of disorder averages is necessary, as a subtraction analogous to that in (29) below will vanish for this case. After coarse-graining both $i$ and $j$ over their respective averaging regions in the neighborhoods of $x$ and $y$, we obtain the correlator of the order parameter $Q$

$G\left(x-y, \tau_{1}-\tau_{2}, \tau_{3}-\tau_{4}\right)=$

$\lim _{n \rightarrow 0} \frac{1}{n(n-1)} \sum_{a \neq b}\left\langle\left\langle Q_{\mu \nu}^{a b}\left(x, \tau_{1}, \tau_{3}\right) Q_{\mu \nu}^{a b}\left(y, \tau_{2}, \tau_{4}\right)\right\rangle\right\rangle$.

$G$ will be found later to behave as the propagator for fluctuations of the $Q$ order parameter field about the mean-field theory, and is directly analogous to a corresponding object in the classical spin glass.

This correlation function can be determined in the tree approximation, from Gaussian fluctuations about the saddle point. To do this we must expand $Q$ about its saddle- point value

$Q_{\mu \nu}^{a b}\left(k, \omega_{1}, \omega_{2}\right)=\beta \delta_{\mu \nu} \delta^{a b}(2 \pi)^{d} \delta^{d}(k) \delta_{\omega_{1}+\omega_{2}, 0} D\left(\omega_{1}\right)$

$$
+\tilde{Q}_{\mu \nu}^{a b}\left(k, \omega_{1}, \omega_{2}\right)
$$

and evaluate correlators of $\tilde{Q}$. Expanding $\mathcal{A}$ to order $\tilde{Q}^{2}$ we can obtain the propagator of the $\tilde{Q}$ field. It is easy to see that when $a \neq b$, this propagator is in fact $1 / M$ times the $G$ correlator (Eqn (22))

$G\left(k, \omega_{1}, \omega_{2}\right)=\frac{M t}{k^{2}+\sqrt{\omega_{1}^{2}+\tilde{r}}+\sqrt{\omega_{2}^{2}+\tilde{r}}}$.

Note that this propagator has a factor $t$ in the numerator and is independent of $\kappa$-the factors of $\kappa$ were placed judiciously in $\mathcal{A}$ to achieve this. FFrom the form of (24) we can deduce that at the critical point $\tilde{r}=0,|\omega|$ scales like $k^{2}$, so the dynamic exponent $z=2$, while for $\tilde{r} \neq 0$ there is a length scale $\xi \sim \tilde{r}^{-1 / 4}$ so that the exponent defined by $\xi \sim\left(r-r_{c}\right)^{-\nu}$ in regime $\mathrm{I}$ is $\nu=1 / 4$.

We can now define the correlation exponent $\eta$. The basic correlation function is $G$, and we define $\eta$ by

$G(k, 0,0) \sim k^{-2+\eta}$

so that $\eta=0$ in mean field theory, as is conventional. In real space, $G$ then decays as $x^{-(d+2 z-2+\eta)}$. Our definition, though conventional in its relation to mean field theory, differs from that used in two recent papers [14,15]. Their $\eta$, which we call $\eta^{\prime}$, is related to ours by $\eta^{\prime}=\eta+z$.

An important susceptibility is related to this correlator. The Edwards-Anderson spin-glass susceptibility $\chi_{s g}$ is given in the paramagnetic phase by

$\chi_{s g}=\sum_{j}\left[\chi_{i j}^{2}\right]$

where

$\chi_{i j}=\int d \tau\left\langle S_{i \mu}(0) S_{j \mu}(\tau)\right\rangle$.

and is analogous to the corresponding object used in the classical theories. After coarse graining we have the expression in terms of $G$

$\chi_{s g}=\int d^{d} x d \tau_{1} d \tau_{2} G\left(x, \tau_{1}, \tau_{2}\right)$.

A second correlator arises upon considering fluctuations (due to the randomness) of the onsite spin correlation function $\left\langle S_{i \mu}\left(\tau_{1}\right) S_{i \mu}\left(\tau_{2}\right)\right\rangle$. 
The second cumulant of these fluctuations can be obtained from a quantum mechanically disconnected correlation function

$$
\begin{aligned}
& G^{d}\left(i-j, \tau_{1}-\tau_{2}, \tau_{3}-\tau_{4}\right) \equiv \\
& \quad\left[\left\langle S_{i \mu}\left(\tau_{1}\right) S_{i \mu}\left(\tau_{2}\right)\right\rangle\left\langle S_{j \nu}\left(\tau_{3}\right) S_{j \nu}\left(\tau_{4}\right)\right\rangle\right] \\
& \quad-\left[\left\langle S_{i \mu}\left(\tau_{1}\right) S_{i \mu}\left(\tau_{2}\right)\right\rangle\right]\left[\left\langle S_{j \nu}\left(\tau_{3}\right) S_{j \nu}\left(\tau_{4}\right)\right\rangle\right] .
\end{aligned}
$$

After coarse graining this becomes another twopoint correlation function of the order parameter, $Q$

$$
\begin{aligned}
G^{d}\left(x-y, \tau_{1}-\tau_{2}, \tau_{3}-\tau_{4}\right)= \\
\lim _{n \rightarrow 0} \frac{1}{n(n-1)} \sum_{a \neq b}\left\langle\left\langle Q_{\mu \mu}^{a a}\left(x, \tau_{1}, \tau_{2}\right) Q_{\nu \nu}^{b b}\left(y, \tau_{3}, \tau_{4}\right)\right\rangle\right\rangle \\
\quad-D\left(\tau_{1}-\tau_{2}\right) D\left(\tau_{3}-\tau_{4}\right)
\end{aligned}
$$

obtained as before by averaging over $i$ and $j$ in neighborhoods of $x$ and $y$. The analog of $G^{d}$ in a classical spin glass is trivial, since $S_{i \mu}^{2}=1$.

The Gaussian approximation for $G^{d}$ can be easily obtained; it is

$$
G^{d}\left(k, \omega_{1}, \omega_{2}\right)=\frac{1}{t^{2}} \frac{G\left(k, \omega_{1},-\omega_{1}\right) G\left(k, \omega_{2},-\omega_{2}\right)}{(1+(u+M v) L(0, k, \tilde{r}))^{2}} .
$$

Note that $G^{d}$ is independent of $t$. Here

$$
\begin{aligned}
& L(\omega, k, \tilde{r}) \equiv \\
& \quad \frac{1}{\beta} \sum_{\Omega} \frac{1}{k^{2}+\sqrt{\Omega^{2}+\tilde{r}}+\sqrt{(\Omega-\omega)^{2}+\tilde{r}}} \\
& \quad \approx \frac{1}{2 \pi} \log \left(\frac{\Lambda_{\omega}}{\max \left(k^{2},|\omega|, \sqrt{\tilde{r}}, T\right)}\right)
\end{aligned}
$$

is a frequency integral.

We can define another exponent for $G^{d}$, in analogy with the random field Ising model, by

$G^{d}(k, 0,0) \sim k^{-4+\bar{\eta}}$

so that $\bar{\eta}$ is also zero in the Gaussian approximation. In real space $G^{d} \sim x^{-(d+2 z-4+\bar{\eta})}$.

Finally, to exhaust the set of different two-point correlators of the $Q$ field, we consider the connected correlation function $G^{c}$, which is the disorder average of the fully connected correlator of 4 spins:

$$
\begin{gathered}
G_{\mu \nu \rho \sigma}^{c}\left(i-j, \tau_{1}-\tau_{4}, \tau_{2}-\tau_{4}, \tau_{3}-\tau_{4}\right) \equiv \\
{\left[\left\langle S_{i \mu}\left(\tau_{1}\right) S_{i \nu}\left(\tau_{2}\right) S_{j \rho}\left(\tau_{3}\right) S_{j \sigma}\left(\tau_{4}\right)\right\rangle_{\mathrm{con}}\right] .}
\end{gathered}
$$

A second susceptibility which can be associated with the spin-glass order is the non- linear susceptibility, $\chi_{n l}$, which is given by

$\chi_{n t}=\int d^{d} x d \tau_{1} d \tau_{2} d \tau_{3} G_{1111}^{c}\left(x, \tau_{1}, \tau_{2}, \tau_{3}\right)$.

The Gaussian approximation for $G^{c}$ is similar to that for $G^{d}$ and will not be given here; we will only note that unlike $G^{d}$, it contains a factor $t$. It is found that, even neglecting the denominators that contain $L, G^{c}$ and $G^{d}$ both vary as $G^{2}$, so are more strongly divergent at long wavelengths than $G$. Discussion of the exponents will be continued below.

\section{RENORMALIZATION GROUP}

We now turn to the effects of fluctuations on the critical properties. We begin with powercounting (dimensional analysis) considerations. We will study the properties of $\mathcal{A}$ under the rescaling transformation

$x^{\prime}=x / b \quad \tau^{\prime}=\tau / b^{z}$

where $z$ is the dynamic critical exponent. The coupling $t$ will play a special role in the following, and it has its own rescaling transformation

$t^{\prime}=t b^{-\theta}$.

The exponent $-\theta$ will be the scaling dimension of $t$ near a fixed point with $t=0$; all fixed points found below in fact will have $t=0$, and $\theta>0$, making $t$ a (dangerously) "irrelevant" coupling. As is conventional, we define the anomalous dimension $\eta$ such that $\eta=0$ in the approximation in the previous section; in RG this means that the coefficient of the $(\nabla Q)^{2}$ term is not rescaled at tree level. This imposes the field rescaling

$Q^{\prime}=Q b^{(d-\theta+2 z-2+\eta) / 2}$.

The exponents $z$ and $\theta$ will be fixed by demanding that the transformations of the $1 / \kappa t$ linear term with time derivatives, and the $1 / t^{2}$ quadratic coupling, are consistent with (37) and the RG equation for the cubic $\kappa / t$ coupling.

It is now a simple matter to determine the tree level rescalings of the remaining couplings (we de- 
termine the rescaling of $\kappa$ from the cubic term):

$$
\begin{array}{ll}
r^{\prime}=r b^{2 z} & \kappa^{\prime}=\kappa b^{(6+\theta-d-3 \eta) / 2} \\
u^{\prime}=u b^{2-z-\eta} & v^{\prime}=v b^{2-z-\eta} .
\end{array}
$$

The full action remains invariant at tree level under these transformations if we choose

$z=2 \quad \eta=0 \quad \theta=2$.

We see then from (39) that $u$ and $v$ are always marginal, while $\kappa$ becomes relevant below $d=8$. As $\theta$ is positive, $t$ is irrelevant and flows towards $t=0$. The marginality of $u$ and $v$ is the reason for the logarithms in the mean field result.

We will not delve into the full one-loop $R G$ equations here; the details are given in [1]. Basically, there are three couplings that control the flows, namely $\kappa, u$, and $U=u+M v$; in this parametrization, the equations are independent of $M$. The main point can be grasped by examining an over-simplified flow equation for $\kappa$ that neglects terms of order $U$ :

$\frac{d \kappa}{d \ell}=\frac{8-d}{2} \kappa+9 \kappa^{3}$.

This has a fixed point at $\kappa^{2}=(d-8) / 9$, in addition to that at $\kappa=0$ whose stablity was analyzed by power counting above. Thus, similarly to the full equations, there is a fixed point for $\kappa$ at $\kappa^{2}>0$ for $d>8$, which can be seen to be unstable, while below $d=8$, there is no fixed point with $\kappa$ real, except for $\kappa=0$ which is unstable. (This is the reverse of the usual situation, for example in $\lambda \phi^{4}$ Landau theory of a ferromagnet, where all positive couplings $\lambda$ flow to zero for $d>d_{u}=4$, and to a stable fixed point value of order $d_{u}-d$ for $d<d_{u}$.) As a consequence, our theory has definite problems below 8 spatial dimensions, and even above 8 , there is the possibility that the bare value of $\kappa$ places us above the fixed point value that separates flows to zero from flows to infinity, rendering the mean field critical theory invalid there too.

\section{SCALING HYPOTHESES}

In spite of the difficulties revealed by the $\mathrm{RG}$ analysis, we may try to formulate a scaling theory for the critical properties, which, by using only general considerations and not the Landau theory, could be valid even at the "true" or strong coupling $(\kappa \rightarrow \infty$, if this continues to mean anything) RG fixed point. Because of the importance of the dangerously irrelevant (DI) variable $t$ in the Landau theory and to gain greater generality, we will assume that such a variable, also denoted $t$ with scaling dimension $-\theta<0$, exists in the scaling theory. We assume that there is only this one DI variable; we expect that the coupling $\kappa$ that is also DI in Landau theory for $d>8$, will not be at the strong coupling fixed point, as in ordinary critical phenomena. The extension to include more DI variables is straightforward, and of course if none are present, one can simply set $\theta=0$. Moreover, it will be assumed that $t$ appears in a similar way as in the Landau theory, which contains a $1 / t^{2}$ term as well as $1 / t$ terms. This means that in each realization of disorder, $t$ plays a role similar to $\hbar$ in quantum field theory (or $T$ in classical statistical mechanics): when it is small, certain types of disorder-induced fluctuations (those directly responsible for determining the local position of the critical point) dominate. (The analogy is not exact because of the internal frequency integrals that can occur even in tree diagrams in $Q$ within Landau theory.)

Defining $G^{d}$ as in Section 3, we have already defined the exponent $\bar{\eta}$ by

$G^{d}(x, \tau, \tau) \sim x^{-(d+2 z-4+\bar{\eta})}$

for fixed $\tau / x^{z}$ at criticality; there is no dependence on $t$ on the right hand side. Thus the dimension of $Q \sim S S$ when the spins are separated in time is $(d+2 z-4+\bar{\eta}) / 2$. Therefore also

$D \sim \tau^{-(d+2 z-4+\bar{\eta}) / 2 z}$.

The spin glass correlator, $G$, (recall (22)) vanishes if $t=0$, so is proportional to $t$ (as $t \rightarrow 0$ ) and thus behaves as

$G(x, 0,0) \sim x^{-(d+2 z-4+\bar{\eta}+\theta)}$.

Comparing with the definition of $\eta, G \sim$ $x^{-(d+2 z-2+\eta)}$ yields

$\theta=2+\eta-\bar{\eta}$.

¿From these we may obtain other scaling relations, e.g. the spin-glass susceptibility $\chi_{S G} \sim$ 
$\left(r-r_{c}\right)^{-\gamma}$ with

$\gamma=(2-\eta) \nu$

and the order parameter $q=\left[\langle S\rangle^{2}\right] \sim\left(r_{c}-r\right)^{\beta}$ with

$\beta=(d+2 z-\theta-2+\eta) \frac{\nu}{2}$.

In general, the usual scaling relations are obeyed, except that hyperscaling involves $d+2 z-\theta$ in place of $d$ for classical critical phenomena whenever the bilocal field is involved (hence the $2 z$ ) and the $\theta$ is due to the DI variable $t$. On the other hand, for a field that is local in time $d$ is replaced by $d+z-\theta$. Thus the free energy density scales as $\left(r-r_{c}\right)^{(d+z-\theta) \nu}$ as $r \rightarrow r_{c}$, and the specific heat at finite temperature $T \rightarrow 0$ at $r=r_{c}$ behaves as $T^{(d-\theta) / z}$; the values $z=2, \theta=2$ yield $T^{3}$ at $d=d_{u}=8$ in the Gaussian theory, as was obtained directly in the infinite-range model [7] and from the mean field theory in [1].

A local variable, which has not been introduced so far, but is important for the present scaling considerations, is the "thermal" operator $\psi(x, \tau)$. This variable couples to the control parameter $r$ that tunes the system across the quantum transition:

$\psi(x, \tau)=S_{\mu}(x, \tau) S_{\mu}(x, \tau)$

in a single replica of the system. For the disconnected correlator of $\psi$ we may define at criticality

$\left[\left\langle\psi\left(x, \tau_{1}\right)\right\rangle\left\langle\psi\left(0, \tau_{2}\right)\right\rangle\right] \sim x^{-\left(d-4+\bar{\eta}_{\psi}\right)}$

(so that the Fourier transform $\sim k^{-4+\bar{\eta}_{\psi}}$; note the correlator is independent of $\tau_{1}, \tau_{2}$ ) so that the scaling dimension of $\psi$ is $\left(d-4+\bar{\eta}_{\psi}\right) / 2$. The connected correlation function then goes as $\sim t$ and so

$$
\begin{gathered}
{[\langle\psi(x, 0) \psi(0,0)\rangle]-[\langle\psi(x, 0)\rangle\langle\psi(0,0)\rangle]} \\
\sim x^{-\left(d-4+\theta+\bar{\eta}_{\psi}\right)} \\
\quad \equiv x^{-\left(d+z-2+\eta_{\psi}\right)}
\end{gathered}
$$

(from $k^{-2+\eta_{\psi}}$ in Fourier space) and hence

$\theta=2+z+\eta_{\psi}-\bar{\eta}_{\psi}$

As usual the dimension of $\psi$ determines that of $r-r_{c}$, and hence the value of $\nu$, through modified hyperscaling, that is

$$
\begin{aligned}
\frac{1}{\nu} & =d+z-\theta-\frac{1}{2}\left(d-4+\bar{\eta}_{\psi}\right) \\
& =\frac{1}{2}\left(d+z-\theta+2-\eta_{\psi}\right) .
\end{aligned}
$$

An interesting rigorous inequality was proved by Schwartz and Soffer [11] for the exponents $\eta_{\psi}$, $\bar{\eta}_{\psi}$ in any system where a local field $\psi$ couples to Gaussian disorder, as is the case for our $\psi$. Extending their proof to the case where the disorder is time independent, we obtain

$\bar{\eta}_{\psi} \leq 2 \eta_{\psi}$

and hence from (51) $\theta \geq 2+z-\eta_{\psi}$. Using (52) this implies

$\nu \geq \frac{2}{d}$

This inequality was proved by Chayes et al. [12]. The present approach appears easier but rests upon the use of a scaling relation to obtain $\nu$.

For the classical random field Ising model, it has been claimed that $\bar{\eta}_{\psi}=2 \eta_{\psi}$ is satisfied as an equality [13]. This would imply that the correlation length at $T=T_{c}$ due to a uniform field $h$ would go as $\xi \sim h^{-2 / d}$. However we find the proof unconvincing, though series results do seem to show that the equality is accurately obeyed in $d=3,4,5$ in that problem.

Since $\psi=S^{2}$ it is tempting to equate the scaling dimensions of $Q$ and $\psi(d+2 z-4+\bar{\eta} \stackrel{?}{=}$ $\left.d-4+\bar{\eta}_{\psi}\right)$ and obtain using (45) and (51)

$\eta_{\psi} \stackrel{?}{=} z+\eta$

However $\psi$ involves bringing spins $S$ to the same time as well as position (and summing over spin indices) and so may have different renormalizations than $Q$. Thus we do not expect this relationship to hold.

We now compare the above relations to Monte Carlo results for $M=1$ in $d=2$ [15] and $d=3$ [14]. Their results are $\nu^{-1} \approx 1.3, z \approx 1.3$ $(d=3)$ and $\nu^{-1} \approx 1, z \approx 1.5(d=2)$. They examined scaling of several susceptibilities, most of which are related to $G$ and hence involve $\eta$ in straightforward ways; however their definition of 
$\eta$ is what we denote $\eta^{\prime}=\eta+z$. In our notation their results are $\eta \approx-0.2(d=3), \eta \approx-1.0$ $(d=2)$. (We have corrected an arithmetical error in the paper by Guo et al. [14]: $\eta^{\prime}=1.1$, not 0.9.) They also examined $\chi_{n}$ which is related to $G^{c}$ (Eqn (35)) and find that its scaling dimension is consistent with the assumption that $\chi_{n l} \sim L^{z+2-\eta}$, with the same $\eta$ as the other susceptibilities.

The numerical results cited so far give no test of hyperscaling, but Guo et al. [14] also studied $D$, obtaining $D(\tau) \sim \tau^{-1.3}$. Using the scaling relations and values of exponents in $d=3$ we ob$\operatorname{tain} \theta \approx 0.0$. This may mean that conventional hyperscaling is obeyed, though because of uncertainties in exponents, a small positive $\theta$ cannot be ruled out. Clearly more work on this point, and tests of other scaling relations, would be welcome. It is interesting that in both $d=2$ and 3 , $\nu^{-1} \approx d / 2$ and this also holds exactly in the $d=1$ model [16] - this raises the question whether the inequalities (53), (54) are saturated; at present we have no argument why this might be so.

\section{METALLIC SPIN GLASS}

Here we add some remarks on the distinctive features found in another spin glass model that we have studied by similar Landau theory techniques, the metallic spin glass [2] (the same model has also been studied by Sengupta and Georges [17], using the techniques of Ref. [7]). In this case the kinetic terms in the action are modified, from $\omega^{2}$ in the preceding models, to $|\omega|$, which represents the long-time persistence of local spin fluctuations in a metal. This change has the effect that the mean field value of $z$ is 4 , and that the $u$ and $v$ couplings are now (dangerously) irrelevant with eigenvalue -2 in the same approximation. The critical fixed point theory is now found to be static, that is it involves only zero frequency fluctuations. In the general scaling scenario, the three 2-point functions of $Q$ have three independent scaling dimensions, and the divergence of the non-linear susceptibility is weakened. In addition, we speculated that additional scaling relations

$z=\nu^{-1}=(d-\theta+2-\eta) / 2$ apply in this case.

\section{CONCLUSION}

To conclude, we have solved, in mean field theory, the problem of the zero-temperature quantum phase transition in the quantum spin glass models defined here. Some questions about the validity of this theory were raised by the $R G$ near the upper critical dimension $d=8$; in particular, we found no extension to non-mean-field exponents below $d=8$. These may indicate that "Griffiths phase" effects are important in determining the critical properties. Nonetheless, our Landau theory approach is of such a canonical nature, that we expect it to be of value in future work on this problem.

\section{REFERENCES}

1. N. Read, S. Sachdev, and J. Ye, Phys. Rev. B 52, 384 (1995).

2. S. Sachdev, N. Read, and R. Oppermann, Phys. Rev. B, (in press).

3. W. Wu, B. Ellman, T.F. Rosenbaum, G. Aeppli, and D.H. Reich, Phys. Rev. Lett. 67, 2076 (1991); W. Wu, D. Bitko, T.F. Rosenbaum, and G. Aeppli, Phys. Rev. Lett. 71, 1919 (1993).

4. W.D. Wu, A. Keren, L.P. Le, G.M. Luke, B.J. Sternlieb, Y.J. Uemura, C.L. Seaman, Y. Dalichaouch and M.B. Maple, Phys. Rev. Lett. 72, 3722 (1994).

5. S. Sachdev and J. Ye, Phys. Rev. Lett. 70, 3339 (1993).

6. D.A. Huse and J. Miller, Phys. Rev. Lett. 70, 3147 (1993).

7. J. Ye, S. Sachdev, and N. Read, Phys. Rev. Lett. 70, 4011 (1993); J. Ye, Ph.D. thesis, Yale University, unpublished.

8. For a review, see, e.g., Spin Glasses, by K.H. Fischer and J.A. Hertz, Cambridge University Press, Cambridge (1991); or, Spin Glass Theory and Beyond, by M. Mezard, G. Parisi, and M.A. Virasoro, World Scientific, Singapore (1987).

9. S. Chakravarty, B.I. Halperin, and D.R. Nelson, Phys. Rev. B 39, 2344 (1989). 
10. A. Chubukov, S. Sachdev, and J. Ye, Phys. Rev. B 49, 11919 (1994).

11. M. Schwartz and A. Soffer, Phys. Rev. Lett. 55, 2499 (1985).

12. A.B. Harris, J. Phys. C 7, 1671 (1974); J.T. Chayes, L. Chayes, D.S. Fisher, and T. Spencer, Phys. Rev. Lett. 57, 2999 (1986).

13. M. Schwartz, M. Gofman, and T. Natterman, Physica A 178, 6 (1991); M. Gofman, J. Adler, A. Aharony, A.B. Harris and M. Schwartz, Phys. Rev. Lett. 71, 1569 (1993) and references therein.

14. M. Guo, R.N. Bhatt, and D.A. Huse, Phys. Rev. Lett. 72, 4137 (1994).

15. H. Rieger and A.P. Young, Phys. Rev. Lett. 72, 4141 (1994).

16. D.S. Fisher, Phys. Rev. Lett. 69, 534 (1992); Phys. Rev. B 51, 6411 (1995).

17. A.M. Sengupta and A. Georges, Phys. Rev. B (in press) (preprint cond-mat/9504120). 